\title{
Correlated Petrographic Analysis
}

\author{
John A. Reffner ${ }^{1}$ \\ ${ }^{1}$ Sciences Department/John Jay College, CUNY, New York, NY 10019, USA
}

This research reports applications of infrared and Raman spectroscopy to correlate microstructure, elemental composition and molecular structure of petrographic sections of rocks. Together, the combination of morphology, optical properties, elemental composition and molecular bonding provide a more complete analysis of a material, and increases our understanding of the processes of its formation and history. Henry Clifton Sorby took up the study of rocks and minerals using a polarized light microscope, and published an important memoir "On the Microscopical Structure of Crystals" in 1858 (Quart. Journ. Geol. Soc.). He pioneered petrography, the study of thin sections of rocks. Sorby also construction and use a micro-spectroscope to analyze the color of materials. Unknowingly, Sorby was the first scientist to practice correlated microscopy; combining microscopy and spectroscopy. Today, we are privileged to use photon and electron microscopes linked to a variety of spectrometers. Each spectrometer provides its unique information on the composition and structure of matter. The scanning electron microscope and microprobe, introduced over fifty years ago, correlate elemental composition with the microstructure of materials. This 'element mapping' is now a routine technology; and is role in materials science is of prime importance. However, these technologies have limited ability when organic and molecular materials are analyzed. Infrared and Raman microprobes provide spectral data which provide details about the molecular structure on the microscopic scale. The unique advantages of molecular micro-spectroscopy are the ability to analyze hydrates, hydrogen-bonded water, and different states of covalent bonding in carbon, nitrates, phosphates, sulfates, silicates and numerous organic and inorganic materials. Polymorphic phases with identical elemental compositions have unique molecular spectra.

The development of an internal reflection objective for infrared microspectral analysis using a diamond internal reflection element (IRE) provides a unique tool to analyze molecular phases observed in petrographic sections. The infrared absorption by carbonates, sulfates phosphates, silicates and other covalently bonded molecular ions strongly absorb min-infrared radiation. Extremely thin sections are required for transmission spectral measurements. Internal reflection methods produce attenuated total reflection spectra (ATR). ATR spectra are independent of sample thickness. Petrographic sections, prepared using standard techniques that date back to Sorby's times may be analyzed with the diamond ATR. Standard petrographic polished sections, without a coverslip, are imaged with visible light through the diamond IRE. The diamond makes contact with the area of interest and the infrared ATR spectrum is recorded.

An example of identifying individual phases of a rock section using ATR infrared spectra is shown in Figure 1. The correlation of microscopical imaging, elemental composition and molecular spectral analysis techniques provides information to better understand the chemistry and structure of materials. Vibrational spectroscopy provides unique information on molecular bonding. In muscovite, the infrared spectrum records the presence of $-\mathrm{O}-\mathrm{H}$ and hydrogen bonded water. This is information is important to understanding the optical properties and thermal behavior of this material. 
References:

1. "On the Microscopical Structure of Crystals" in 1858 (Quart. Journ. Geol. Soc.vol. xiv, pp. 453-500)

Diamond ATR Infrared Spectra of Mineral Phases in Petrographic Section
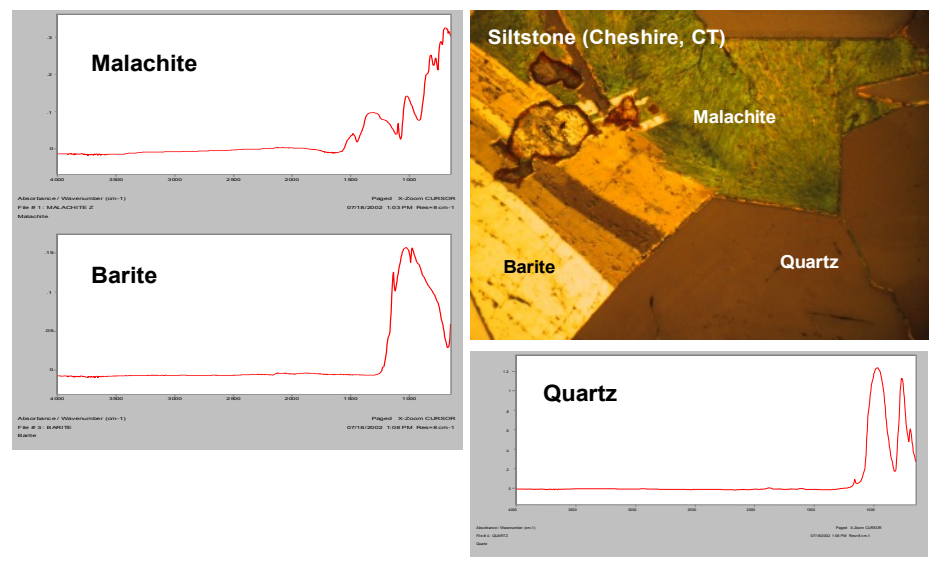

Figure 1. The collection of ATR mid-infrared spectra of rock sections is made using an FT-IR microprobe and a diamond internal reflection objective. The molecular bonding of the mineral phases is determined by comparison to standard spectral data libraries.

\section{$2 \mathbf{v}=+\quad$ Optic Sign +}
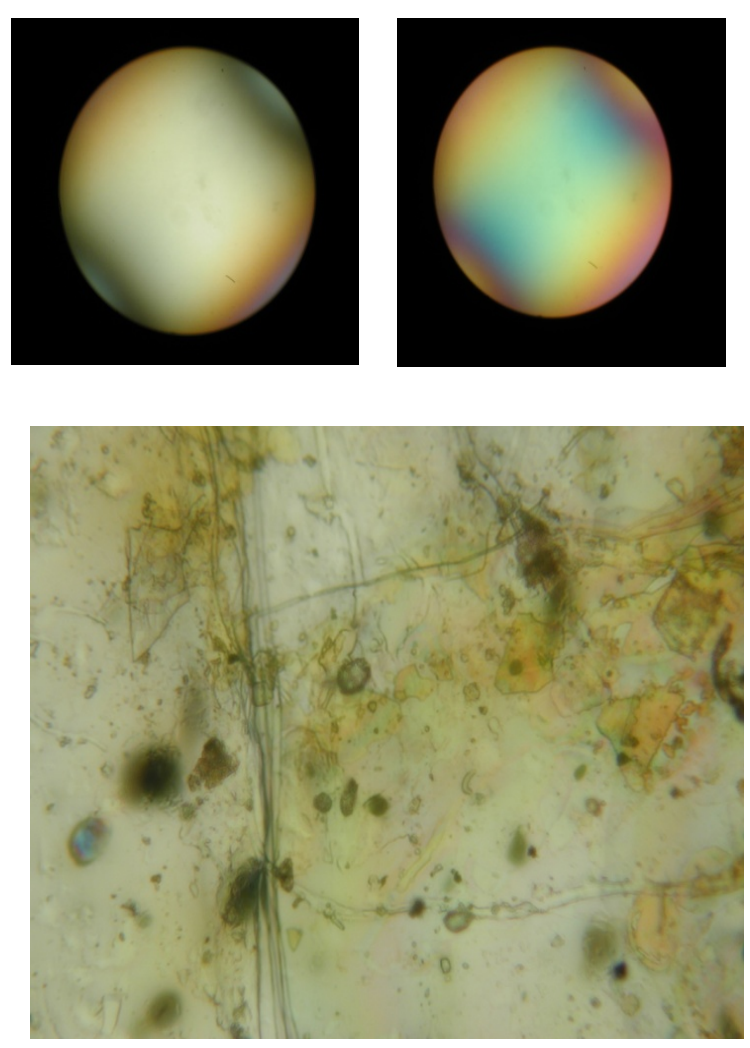

Figure 2. Mica is a group of sheet silicates composed of aluminum and potassium with hydroxyl or fluorine and magnesium, iron, or lithium.

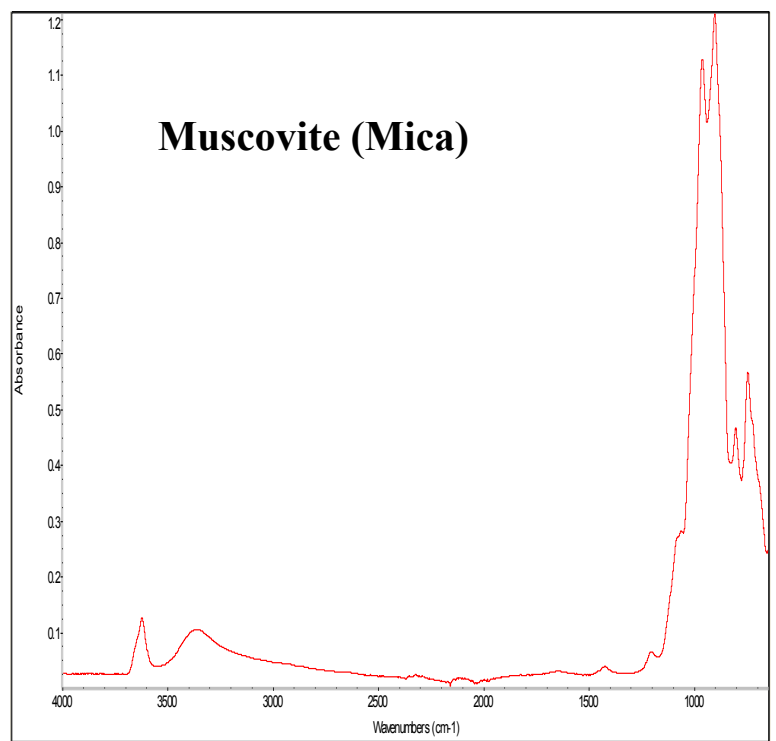

